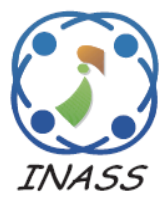

\title{
ABNC: Adaptive Border Node Clustering Using Genes Fusion Based on Genetic Algorithm to Support the Stability of Cluster in VANET
}

\author{
Ronald Adrian $^{1,2} \quad$ Selo Sulistyo $^{1 *} \quad$ I Wayan Mustika $^{1} \quad$ Sahirul Alam $^{1}$ \\ ${ }^{1}$ Department of Electrical Engineering and Information Technology, Faculty of Engineering, \\ Universitas Gadjah Mada, Yogyakarta, Indonesia \\ ${ }^{2}$ Department of Electrical Engineering and Informatics, Vocational College, \\ Universitas Gadjah Mada, Yogyakarta, Indonesia \\ * Corresponding author’s Email: selo@ugm.ac.id
}

\begin{abstract}
The clustering method in the vehicular ad-hoc network provides an opportunity for a cluster head to improve the network connections, but it still remains a problem in the border cluster. The vehicles, in view of their position in the slices of two clusters, receive a weak signal from the cluster head. The intersection area causes a throughput decrease for cluster members. To provide the network connection in the border cluster, we, in turn, proposed an adaptive border node clustering by utilizing the combination of K-Medoids algorithm, modified Genetic Algorithm and modified Tabu Search. We ameliorated the recent model in Enhanced Model of Weighted K-Medoids Clustering Algorithm by adding the fusion process to the best gene pieces into one individual temporary. The effects of this fusion were on the production of a temporary cluster that can make the main cluster environment more stable. This temporary cluster was adapted to the position of the two closest main cluster heads. Our model was found able to increase throughput and to keep the stability of cluster members at any velocities. The increase in throughput represents an improvement parameter in the network quality of service (QoS). We achieved the overall throughput at $93.97 \%$ (throughput vs cluster member) compared with the previous methods and the cluster member stable at around 25 vehicles (cluster member vs transmission range) in varied conditions.
\end{abstract}

Keywords: Clustering, Genetic algorithm, K-medoids, Tabu search.

\section{Introduction}

Vehicular ad-hoc network (VANET) has an important part in future transportation technology. In it, many vehicles are able to communicate with each other through the $802.11 \mathrm{p}$ standard [1]. This communication provides vehicle information, such as speeds, locations, and proximities. Also, it confirms the safety and convenience for driver. If there is an accident in front of the vehicle, it can be quickly informed to others, so it can reduce the possibility of the pileup. This information must be transferred as soon as possible using a reliability connection. Some algorithms have been compared in terms of this convergence time comparison [2]. It also requires a good network infrastructure and a suitable method. VANET consists of two connection types in its network infrastructure. Vehicles can be connected using V2V (vehicle to vehicle connection) or RSU (Road-Side Unit) to improve the visibility data from others [3].

Vehicle grouping is an important process to improve the quality of VANET network connections. In an adjacent clustering process, it will create a cluster border; as a consequence, the connection stability is interrupted. An evaluation of this cluster border has been investigated using a combination of the Genetic Algorithm and Tabu Search methods [4]. These algorithms detected the similarity of vehicles in the border cluster area with the cluster head. This method aimed to decide which clusters would be used by vehicles along with the border cluster. Each vehicle on the border cluster will have two choices: staying in the initial cluster or moving to a new cluster that matches the characteristics of the vehicle. 
The border cluster phenomenon becomes an interesting area to be resolved [4]. Though only a few researchers concern in this area, there is still room for improvement that can be extracted in this border cluster. In this paper, we introduced a new method in border cluster mining by means of the combination of three algorithms: K-Medoids, Genetic Algorithm, and Tabu Search.

In our novel, we modified the crossover of the Genetic Algorithm process to create an adaptive border node cluster. We separated the best gene of the parent chromosome using the similarity value without crossover. It is slightly different from the previous method based on the original Genetic Algorithm. In original Genetic Algorithm, the parent's and the children's genes will be combined into one chromosome [4]. They didn't use the temporary cluster to increase the stability of main cluster. In our proposed method, the best children's gene will be collected into one group without any influence of parent. It depends on the similarity value in certain time. Furthermore, it leads a temporary cluster based on a specific condition and gives a significant impact on cluster stability especially in throughput. The throughput shows the stability under any condition. We compared the results of the study with the An Adjusted K-Medoids Clustering Algorithm for effective stability in vehicular ad hoc networks (AKCA) [7] and Enhanced Model of Weighted K-Medoids Clustering Algorithm (EWKCA) [4] methods as the recent methods running in the cluster problems.

The rest of this paper is structured as follows. Section 2 is designed to present the recent related work and Section 3 presents the theoretical support of the proposed method. Section 4 and Section 5 respectively present our proposed method, ABNC and discussion and comparison about the result with the previous method. Lastly, Section 6 presents the conclusion of this research.

\section{Related Works}

Clustering is the mature method in VANET. Clearly, it grouped the vehicles to improve network quality [5]. Few researchers have explored the improvement of the clustering method to achieve a stable cluster and foreclose the surrounding research gap.

In 2018, a comprehensive survey on clustering methods in VANET was conducted by Cooper. They presented the genealogical algorithms that had been used in research at VANET. The core of this survey was on the cluster head selection technique in which the cluster head would take the network feeder in cluster formation. The survey covered most of the studies from 1987 to 2016 [6]. In this section, we add the information of some recent research that will serve as the evaluation of our proposed method.

The Enhanced Model of Weighted K-Medoids Clustering Algorithm (E-WKCA) is the first paper that discusses border cluster issues. It was conducted by Hajlaoui in 2019 [4]. Their proposed method used the combination of metaheuristic algorithms. It covered the Genetic Algorithm and Tabu Search. They introduced the weighting in clustering. The weighting covers the direction value, speed level, and proximity to the cluster head. The improvements to this method were named as the adjusted K-Medoids clustering algorithm for effective stability in vehicular ad-hoc networks (AKCA) [7].

Genetic Algorithm and Tabu Search are the main components in border node evaluation. Genetic algorithms are also used on backup routes on the Mobile Ad Hoc Network (MANET) [8]. Evaluations are carried out to determine which vehicles on the border cluster will continue to join the old cluster or move to the new one. This condition occurs when the vehicle in the cluster intersection area can be easily detected for instance in a Wi-Fi adapter on a laptop that has just been turned on in which many Services Set Identifiers (SSID) on the system can then be seen.

The concept of similarity value is used as the main engine in the evaluation process using Genetic Algorithms. In the Genetic Algorithm, there is a step for crossover between chromosomes. The crossover uses a similar analogy approach to children and parents. Similarity Value includes vehicle speed, closeness to the cluster head and vehicle direction. The speed of this vehicle is compared to the speed of the cluster head at a certain time. Getting a closer margin will also bring about a high level of similarity. The direction of the cluster member is compared with the direction of the cluster head. The lifetime of vehicles will have a longer duration than joining the cluster in the opposite direction.

In the first quarter of 2019, Hajlaoui et al. came with An Adjusted K-Medoids Clustering Algorithm for effective stability in vehicular ad hoc networks (AKCA) [7]. It can solve problems by means of routing on VANET. Clusters are created by AKCA have good stability. AKCA excels in terms of QoS value. In this study, we learned K-Medoids clustering in AKCA to build a new stable cluster. The concept of similarity value was used as the main basis in making clusters using K-Medoids. This similarity value included speed, distance to the cluster head and direction. All these parameters were compared with the cluster head and the cluster members were grouped based upon the level of similarity value with 
the cluster head. AKCA has used the K-Medoids algorithm in cluster formation [7]. With the use of centroid point analogy as a $\mathrm{CH}, \mathrm{AKCA}$ performs well in cluster formation. The application of AKCA has not considered the border clusters phenomenon although it is the latest method in VANET clustering.

The first border cluster problem can be solved using the E-WKCA method [4]. The strength of EWKCA is the use of a Genetic Algorithm with the similarity value approach. Their proposed method is an implementation of the original genetic algorithm in the new problem in the border cluster. Although it is interesting approach, it deficient to provide the throughput stability in varied cluster sizes because the vehicles in the border area received the weak signal from the cluster head. It is caused by the grouping process using an original Genetic Algorithm. The main engine of the Genetic Algorithm is the crossover. the original crossover process involves cutting and merging genes between the child and the parent. The comparison of the original crossover and our proposed method in gene fusion will be further discussed in the next section.

The impact of vehicle grouping is the disposal of network resources. E-WKCA is powerful when the cluster size tends to be fixed. To fill the gap, we improve their method with the crossover modification. We use the concept of cutting off the best child genes and grouping them together without crossbreeding between child and parent. Consequently, our method may cut the genetic algorithm process. It also produces stable throughput in overcoming the problem of border clusters under varied cluster size.

Moreover, the double cluster head can be also a solution for border node problems. However, its use is less effective in the very dynamic cluster conditions. This model requires an accurate estimation in that the process of selecting the second cluster head can disrupt the system stability [9]. The error in selecting two cluster heads in one cluster consequently will confuse the determination of its members.

The determination of cluster size in VANET may use a bio-inspired algorithm [10]. The latest algorithm in the bio-inspired algorithm is moth flame optimization (MFO) invented by Mirjalili in 2015 [11]. The use of MFO was firstly carried out by Shah et al. in which it was named CAMONET [12]. This algorithm aimed to find the optimal cluster size on VANET. It ignored the phenomenon of border clusters, although this algorithm had the best convergence time among previous bio-inspired algorithms such as particle swarm optimization and bat algorithm [2]. A comprehensive study of the bio- inspired algorithm told that PSO is the forerunner of the other intelligent swarm algorithm such as bee foraging optimization (BFO) and ant colony optimization (ACO) in VANET [10]. Moreover, a method in cluster quality improvement introduced in 2019 used the coalitional game technique with an aim to improve the quality of the signal to noise ratio (SNR) and channel capacity by using the Shannon theorem [13]. Coalitional game is applied in a vehicle to vehicle $(\mathrm{V} 2 \mathrm{~V})$ connection by using the analogy of interaction between a set of players who attempt to form a group or coalition to reinforce their standing in the game [14].

The aforementioned related works drive to the new problem and several techniques which adopted in the clustering method. The border cluster becomes a challenges topic that must be resolved. It may decrease network QoS, especially throughput.

\section{Proposed method}

An intersection area in the border cluster gives a new concern in the stability of the cluster. The further explanation can be found in the next subsection. In this section, we provide the main information about our proposed method.

\subsection{Problem description}

In spite of clustering is an established method in ad-hoc network communication, especially on VANET, it leaves a problem in the intersection area in border clusters. An intersection area is formed when there are two or more adjacent clusters. This can be detected by every vehicle in this area. The detection process is very easy with a signal strength comparison. If they detect the same signal strength, they are in the intersection area. The same signal strength will confuse the vehicles in that area to choose which cluster head will be followed. This problem contributes to throughput decrease in cluster members. Overall, it will cause QoS to be disrupted. A new clustering scheme proposed in order to countermeasure this problem. Vehicles on this border cluster can be evaluated more deeply with an aim to get a stable cluster on VANET. Fig. 1 shows the phenomenon in the intersection cluster.

\subsection{Experimental set-up}

Table 1 presents the simulation parameters used in this research. We created a simulation using the Simulation of Urban MObility (SUMO). In this software, we simulated the real mobility model with 


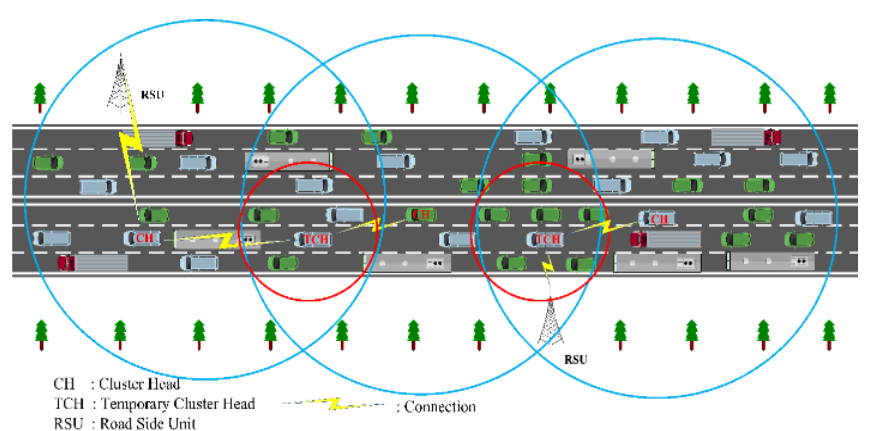

Figure. 1 Road simulation

Table 1. Simulation parameters

\begin{tabular}{|c|c|}
\hline Parameter & Value \\
\hline Road length & $1000 \mathrm{~m}$ \\
\hline Number of Lanes & 6 \\
\hline $\begin{array}{c}\text { Vehicle } \\
\text { Traffic Flow }\end{array}$ & $\begin{array}{c}5000 \text { vehicles } \\
\text { per hour }\end{array}$ \\
\hline Road Type & Highway \\
\hline Vehicle Types & $\begin{array}{c}\text { City Car, SUV } \\
\text { Bus and Truck }\end{array}$ \\
\hline Simulation Time & $100 \mathrm{~s}$ \\
\hline Transmission Range & $300 \mathrm{~m}$ \\
\hline Vehicle Movement & Random \\
\hline Maximum Lane Speed & $120 \mathrm{kph}$ \\
\hline $\begin{array}{c}\text { Gap between } \\
\text { Vehicles }\end{array}$ & $1.5 \mathrm{~m}$ \\
\hline
\end{tabular}

varied conditions. The output file from this software would be further analyzed using the proposed method.

\subsection{Main concept}

The main concept in this research was to develop E-WKCA by using algorithm modification to cope with the problems in the border cluster. The method we proposed is called adaptive border node clustering (ABNC). This adaptive method was formed when there was an intersection cluster. This intersection area can occur either for a long time or for a short time.

Our proposed method could adjust by automatically detecting the border area of the cluster and form or join the main cluster in accordance with the conditions at that time. The purpose of this method was to make the cluster more stable in speed and any number of members.

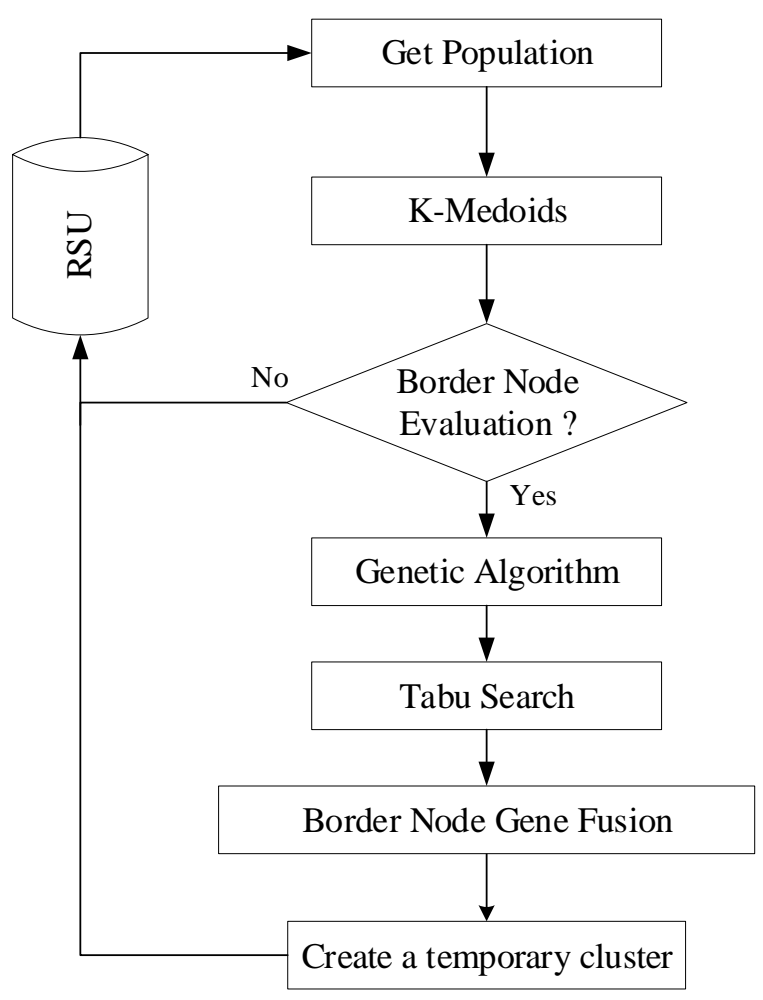

Figure. 2 Flowchart of ABNC

\subsubsection{Getting population}

The initial population is the first step in the Genetic Algorithm to get the population. In this step, we determined the genes and chromosomes. The gene represented a vehicle with its attributes and a chromosome containing several genes. Each gene represented one vehicle in a cluster. Whereas, one chromosome was a representation of a cluster. We got this population data from a simulation of vehicle movements using SUMO for 100 seconds of the experiment.

\subsubsection{K-medoids}

K-Medoids is a common clustering algorithm in VANET. It has a good ability in centroid selection. The selected centroid will act as the cluster head responsible for providing sufficient network resources to its members. In this research, we run the K-Medoids with the following procedure:

a. Determining the number of clusters and their bounds.

K-Medoids is the preliminary step in VANET clustering. In this process, we used the vehicle's position $(\mathrm{x}, \mathrm{y})$ in determining the proximity $(\Delta P)$ among the cluster head and its members. It is shown in Eq. (1). The clustering process improvement was carried out in the next step. 


$$
\Delta P=\sqrt{\left(x_{1}-x_{2}\right)^{2}+\left(y_{1}-y_{2}\right)^{2}}
$$

An original clustering method requires a fixed number of clusters before creating a cluster. Now, it powerfully adapts in dynamic conditions. As in VANET, cluster sizes can change at any time depending upon the speed, density, and direction.

To determine the number of clusters, we use the Eq. (2).

$$
K=\left(\frac{L}{\sum_{i=1}^{n} T R_{i}} \cdot n T r\right)
$$

The clustering must consider the number of clusters and the transmission range from the cluster head candidate. Normally, the transmission range reaches 300 meters. Eq. (2) is used in determining the number of clusters where $\mathrm{K}$ refers to number of clusters, $\mathrm{L}$ is the length of the highway, and $T R_{i}$ is the transmission range.

\section{b. Cluster Heads Generation}

The cluster head is chosen based on its position. It begins by selecting a cluster head that is in the middle position. It is continued by selecting a cluster head with a combination of several important parameters to get the best cluster head.

\section{c. Building the Cluster}

The latest cluster formation method used the concept of similarity value [4]. We still maintain this technique. Eq. (3) used to classify vehicles based on the similarity of properties to the selected cluster head.

$$
S V=\left(w_{1} \cdot D\right)-\left(w_{2} \cdot \frac{\Delta S}{\max (S)}\right)-\left(w_{3} \cdot \frac{\Delta P}{\max (T r)}\right)
$$

Where $D$ is the vehicle direction $(0 / 1), 0$ is the same direction with $\mathrm{CH}$ and otherwise. Then, $\Delta S$ is deviation of speed to the $\mathrm{CH}$. The proximity to $\mathrm{CH}$ is $\Delta P$. The last, $w_{1}, w_{2}$ and $w_{3}$ are the weighting of $D$, $\Delta S$, and $\Delta P$.

\section{d. Cluster Head Switching}

Cluster head switching is used by the existing cluster head to delegate its privilege as cluster head to another. It depends on varied condition as shown in Eq. (4).

$$
W=a_{1}(\Delta D)-a_{2} \Delta S+a_{3}(T r)-a_{4}(N D F)
$$

Where, $a_{1}, a_{2}, a_{3}$ and $a_{4}$ respectively refer to the weighting of $\Delta D, \Delta S, T r$ and NDF (node disconnection frequency)

\subsubsection{Border node evaluation}

Every cluster that intersects with another cluster will definitely make an overlap area. The border node is the vehicle that runs in the border cluster. It can be a burden on the main cluster if no further evaluation. The evaluation process on the E-WKCA uses Genetic Algorithms and Tabu Search to determine which border nodes will remain on the main cluster. The evaluation process we proposed was somewhat different. We separated the genes at the border node from the parent. Then, we grouped these genes with genes similar to the similarity value approach. This similarity value was in the theoretical support.

\subsubsection{Genetic algorithm}

Genetic Algorithm is an algorithm based on parental marriage to get the best children. Generally, parents will inherit superior qualities to their children. This process occurs after going through a crossover between parent genes. Overall, the Genetic Algorithm has three important components: selection, crossover and mutation [15].

Parents $\left(P_{1}\right.$ and $\left.P_{2}\right)$, in a simple crossover, produce two ancestries $\left(C i_{1}\right.$ and $\left.C i_{2}\right)$. We may assume that the parent is $P_{1}=\left[P x_{1}, P x_{2}, . ., P x_{n}\right]$ and $P_{2}=\left[P y_{1}, P y_{2}, . ., P y_{n}\right]$. Then, $r$ is a discrete random number. The range of this number from 1 until the length of vector $\mathrm{x}$ or $\mathrm{y}$. Therefore, the relation of $u$ and $v$ can be interpreted as representing descendants of $C i_{1}$ and $C i_{1}$.

$$
\begin{gathered}
u_{i}=\left\{\begin{array}{cc}
P X_{i}, & i<r \\
P y_{i}, & \text { otherwise }
\end{array}\right. \\
v_{i}= \begin{cases}P X_{i}, & i<r \\
P y_{i}, & \text { otherwise }\end{cases}
\end{gathered}
$$

The value of random number $r$ is often referred to as the intersection point. This value is used as a determination of what gene to crossover. The gene represents a vehicle in the road. For example, there are 8 vehicles therewithal their parameters. It is further analysed with $r=3$. It means the crossover 
starting from the 4 th gene from the $P_{1}$ chromosome will be exchanged for genes from the $P_{2}$ chromosome. Actually, this process is swapping the vehicle parameters to obtain a similar value among them. Whereas in arithmetic crossovers, offspring are produced by the linear combinations of parent vectors. For the continuous values like in VANET, it can be written mathematically with the terms $\lambda_{1}+\lambda_{2}=1$. Eq. (7) and Eq. (8) show the crossover schemes in VANET. $\mathrm{Cr}$ is the chromosome from the crossover result.

$$
\begin{aligned}
& C r_{1}=\lambda_{1} P_{1}+\lambda_{2} P_{2} \\
& C r_{2}=\lambda_{2} P_{1}+\lambda_{1} P_{2}
\end{aligned}
$$

In this research, the binary numbers are used to represent the vehicle values $(D, \Delta S$, and $\Delta P)$. These values deputize as continuous values. It is important to convert the continuous value into a binary value. It will represent the genes into a chromosome. After processing using binary numbers, the results are returned to decimal values with the Eq. (9). This conversion must be under in the origin values. The barrier must be defined as an upper bound $(U B)$ and lower bound $(L B)$.

$$
x=L B+\frac{U B-L B}{2^{q}-1} \sum_{k=0}^{q} 2^{k} b_{k}
$$

The linked clustering process using K-Medoids still has some weaknesses with its adaptation to a border cluster system. Therefore, it needs to be optimized using Genetic Algorithms. This algorithm puts forward the concept of the similarity of traits between children and parents. We would present a detailed improvement in the next subsection to make up the original Genetic Algorithm that has been used in recent research.

As our focus in the border cluster, we used an operator to take over the leader in a cluster. The task of this operator was to act as the temporary cluster head responsible for providing temporary network connections to vehicles in the intersection area. This selection process only took place when there was an intersection cluster. If there was no longer an intersection cluster, the operator would be disappeared. Then, the operator will join the main cluster.

\subsubsection{Tabu search algorithm}

Tabu Search is a metaheuristic optimization algorithm. It is used in the process of finding the best

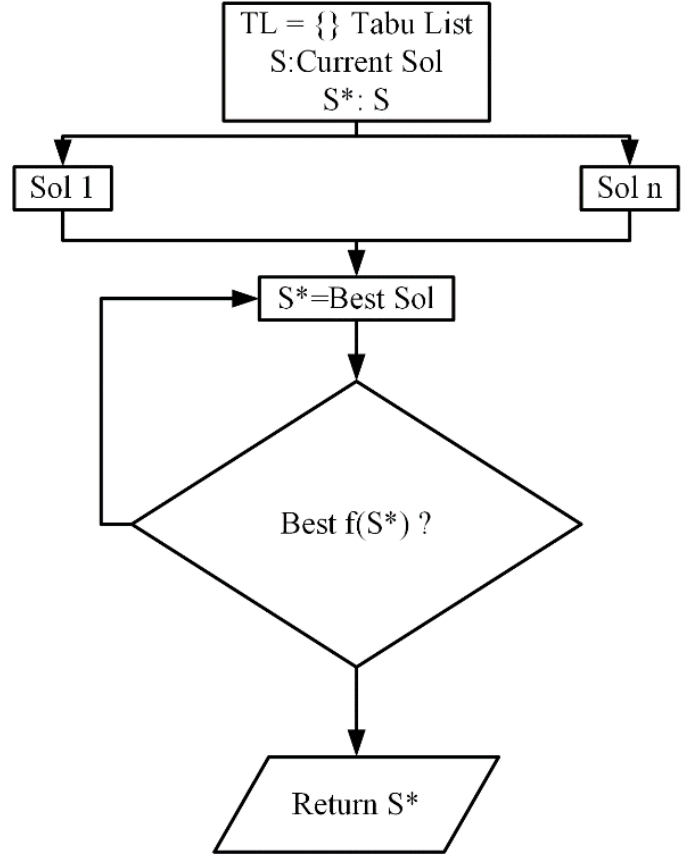

Figure. 3 Flowchart of Tabu search

value. Tabu Search has a very simple concept that is by comparing the candidate's score to the best value on each iteration. The best value here will be used as the final grade [16]. As an initialize, the values from the Genetic Algorithm will act as an initial solution (i) in Tabu Search array $(S)$. It is updated as $\mathrm{S}^{*}=\mathrm{S}$ and $\mathrm{k}=0$. The value of $k$ always is incremented $(k=k+1)$ to produce the best value of fitness function.

We modified the Tabu Search process on the EWKCA. The Tabu Search process we proposed had a simpler flow by not ignoring the goal of finding the best solution value. The Tabu list was obtained from the results of Genetic Algorithms. Then, it was used as input data on the Tabu Search. This process aimed to find the best cluster head that could affect cluster formation. This process is illustrated in Fig. 3.

\subsubsection{Border node gene fusion}

It is the main concept proposed in this study. The idea of gene fusion is based on research in medicine to treat tumor pain [17]. Gene modification in the Genetic Algorithm aims to produce the best genes. In general, Genetic Algorithms crossover using chunks of genes on chromosomes and exchange them for chunks of genes on other chromosomes. Our proposed method is to combine the best gene pieces with the other best gene pieces. This process can produce chromosomes with the best genes quickly as shown in Fig. 4. This best chromosome will later be used as the basis for making temporary clusters. 


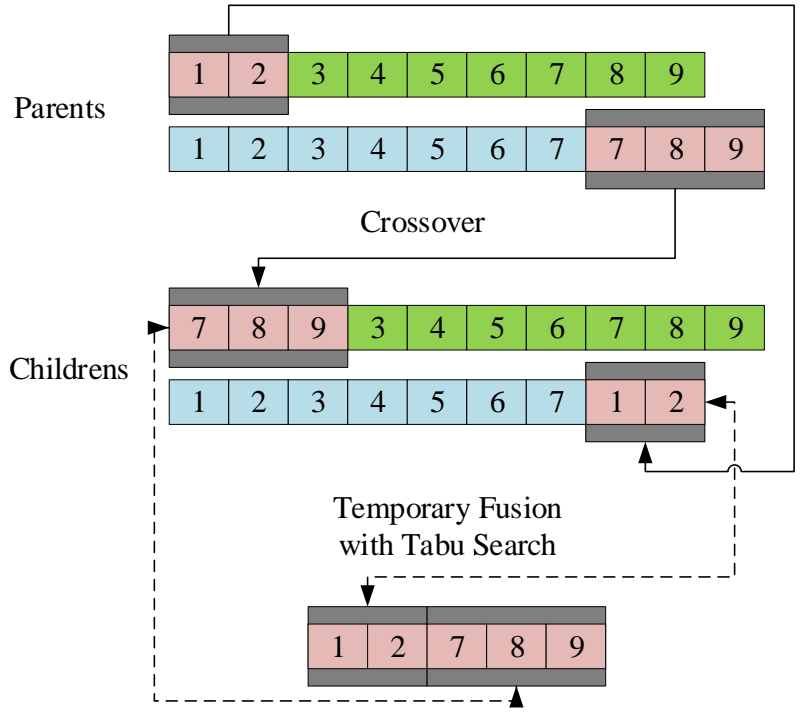

Figure. 4 Adding a fusion in genetic algorithm

A set of genes is a chromosome content as shown in Eq. (10). The gene represented vehicle identities like direction, proximity, and speed. The chromosome represented the cluster of several vehicles.

$$
\operatorname{Cr}=\left[g_{1}, g_{2}, \ldots, g_{n k}\right]
$$

Where, $g_{i}$ is a gene value of the $i^{\text {th }}$ and $n k$ is the problem dimension. If the vehicle detects two connections in the same time, it runs the processes as follow:

$$
\begin{gathered}
{[(x, y), \Delta S, \Delta P] \in g_{i}} \\
g_{p} \in\left(c_{1} \backslash c_{2}\right) \text { or }\left\{g_{p} \in c_{1} \mid g_{p} \notin c_{2}\right\} \\
g_{s}=c_{1} \Delta c_{2} \text { or }\left(c_{1} \backslash c_{2}\right) \cup\left(c_{2} \backslash c_{1}\right) \\
g_{s} \in\left(c_{1} \cap c_{2}\right) \\
c_{1} \cap c_{2}=\emptyset \\
C r_{\text {new }}=\left[g_{s 1}, g_{s 2}, \ldots, g_{s n}\right]
\end{gathered}
$$

Where, $g_{p}$ is the primary genes (parent), $g_{s}$ is the secondary genes (best genes), $g_{s n}$ is the collection of secondary genes (offspring), $c_{1}$ is the first cluster and $c_{2}$ is the second cluster. Lastly, $C r_{\text {new }}$ is the final offspring of the Genetic Algorithm.
The result is driven by Eq. (17) in every process to achieve the best solution. Usually, it is called a fitness function.

$$
\begin{gathered}
F=\sum_{\mathrm{i}=1}^{\mathrm{n}}\left(\left(w_{1} \cdot D\right)-\left(w_{2} \cdot \frac{\Delta S}{\max (\text { speed })}\right)-\right. \\
\left.\left(w_{3} \cdot \frac{\Delta P}{\max (t r)}\right)\right)
\end{gathered}
$$

\subsubsection{Temporary cluster}

A temporary cluster is a product of the proposed method. It is depicted using a red circle as shown in Fig. 5. This circle indicates the range of signals generated by the temporary cluster head. This signal range can be adaptive by using power control. This power control is adaptive to the distance between the temporary cluster head and the main cluster head. This is important so as not to cause a new intersection area. This power control model is shown in Eq. (4).

$$
R P C=\frac{A D 1}{A D 1+A D 2} \cdot R C H
$$

Where, $R P C$ is the new connection range with the power control approach, $A D I$ is the distance between the cluster head temporary and main front cluster head and $A D 2$ is the distance between cluster head temporary and main behind cluster head. RCH, meanwhile, is the maximum connection range of cluster head. The maximum value is 300 meters.

\section{Results and Discussion}

We compared the results with the AKCA [7] and E-WKCA [4] as the previous method in VANET clustering. We show three important measurement parameters in VANET stability including cluster size, cluster head duration and throughput.

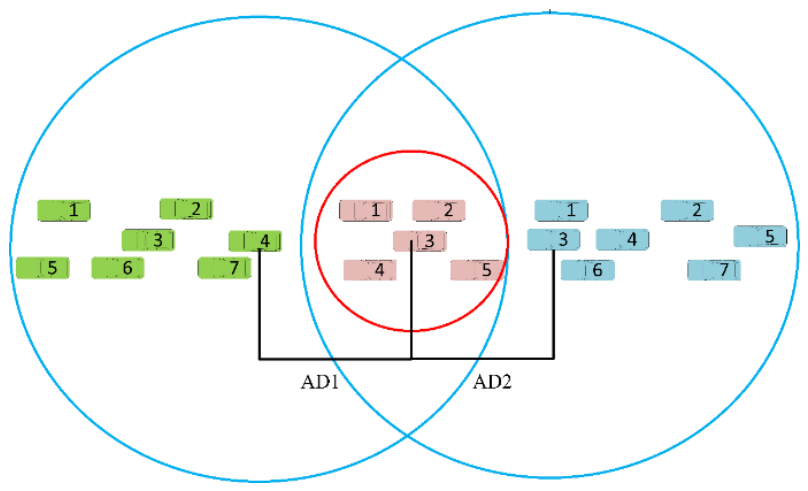

Figure. 5 Clustering model 


\subsection{Cluster size}

Cluster size is one of the parameters of cluster stability. It represents the number of users that have access to a wireless access point. The more users, the less the bandwidth each user obtained. However, the stability of the number of clusters in the value of the transmission range will affect the fairness of network resources.

In Fig. 6, ABNC provides advantages in terms of keeping the cluster size always the same in varying transmission range conditions. This is important because, with the same cluster size, the throughput result will also be stable.

\subsection{Throughput}

Throughput is one of the network qualities of service (QoS) guarantee in VANETs communication [18]. The measurement of throughput is inversely proportional to distance [19]. Vehicles at great distances from the cluster head will have a small throughput. Likewise, the cluster head far away from the roadside unit also has a small output.

The next result is shown in Fig. 7. This demonstrates the throughput of the ABNC method compared to the previous methods. ABNC is very strong in producing a stable output. Irrespective of the cluster member, the throughput will be stable.

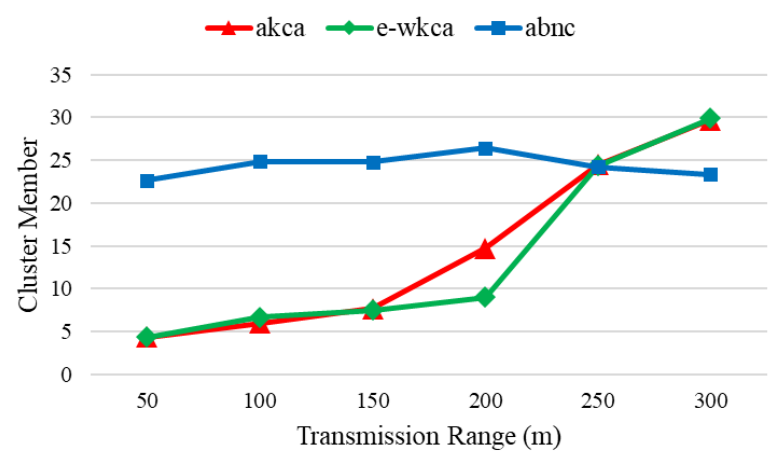

Figure. 6 The number of cluster member for varying transmission range

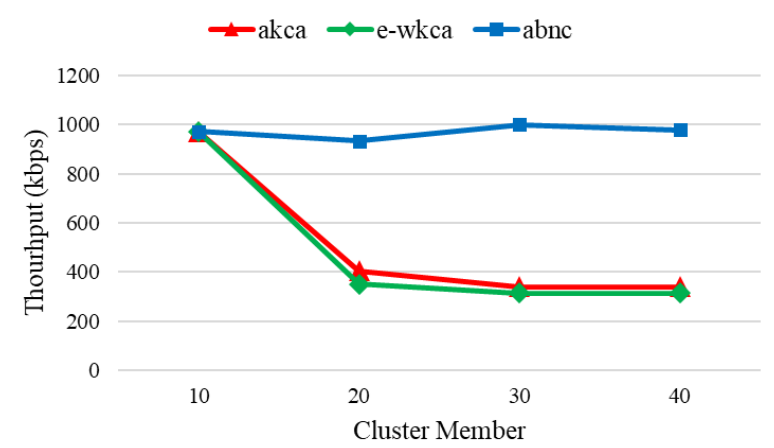

Figure. 7 Throughput of cluster head for cluster member
Cluster size which tends to remain at any transmission range has some advantages to maintain the closeness of the distance between vehicles in a cluster. This cluster will tend to have a fixed size. The fixed distance between vehicles at a fixed cluster size will result in a stable throughput under any circumstances.

$$
\text { throughput }(\%)=\frac{\overline{a_{i}} \overline{b_{i}}}{\overline{b_{i}}} \cdot 100 \%
$$

We use Eq. (19) to calculate the throughput improvement compared with the last method. Where, $\overline{\mathrm{a}_{\mathrm{i}}}$ is the average throughput from our proposed method and $\overline{b_{i}}$ is the average throughput from previous methods (AKCA and E-WKCA).

\subsection{Cluster head duration}

To measure the cluster head duration, we needed to observe the behaviour of the cluster head every second. Vehicles have different speeds when driving on the highway even though most of them have similarities to another. The difference in the similarity level of speed will affect the stability of the cluster system. The cluster head duration is the key component in stable cluster determination.

In Fig. 8, ABNC also provides the same cluster head duration as the previous method. This relates to the stability of the cluster system. In the process of clustering, the selected cluster head has a long duration of time. This method is not inferior to the previous methods. On highway, all vehicles will move at high speed. The uniformity at high speed makes the cluster head duration increase at high speed.

Cluster formation time is the time needed to create a cluster. This time is calculated from the first time the system runs until the cluster is formed. The process of taking this value is carried out on each method to be compared.

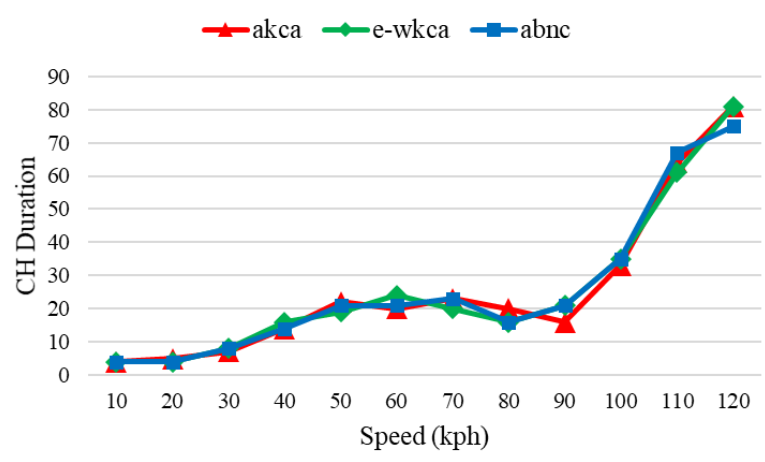

Figure. 8 Cluster head duration for varying speed 


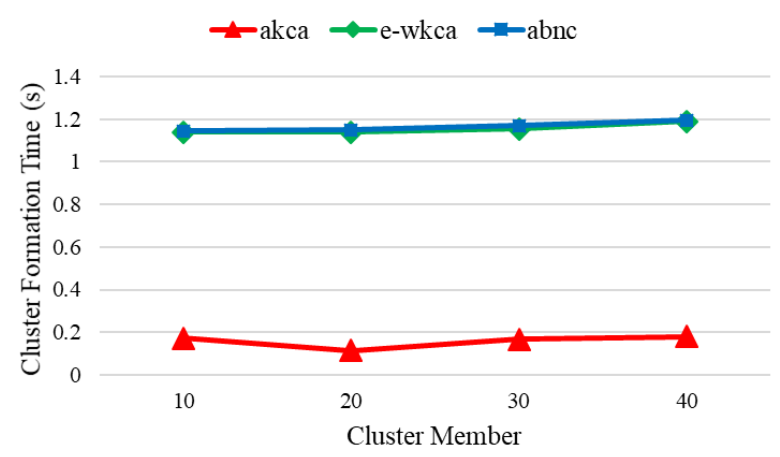

Figure. 9 Cluster formation time

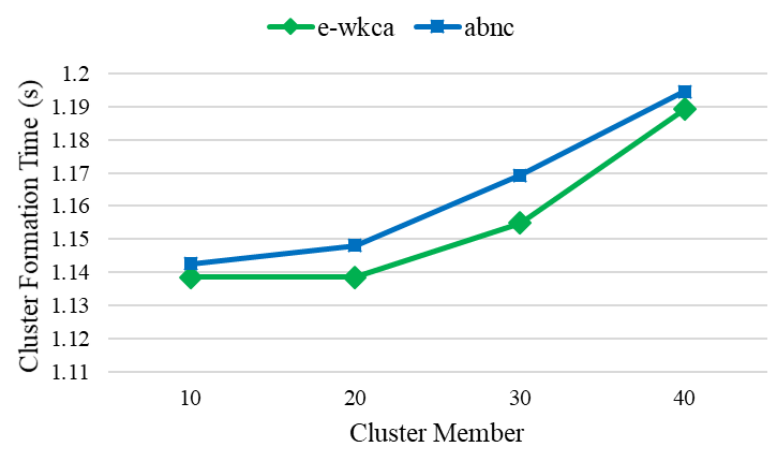

Figure. 10 The detail comparison of cluster formation time

In Fig. 9., AKCA has the best time of cluster formation. It is influenced by using of K-Medoid algorithm only in the clustering scheme. The use of simple algorithms combination will speed up the computing process. Moreover, AKCA does not consider border cluster issues so the complexity is reduced. It has an impact to minimize the computation time.

The fair comparison of the cluster formation time involves the E-WKCA method which also regarding the border clusters issues. The combination of algorithms used in this method consumes longer time. Likewise, our method requires almost the same time as the E-WKCA. However, the method we propose has a stable throughput and cluster size compared to the E-WKCA. In Fig. 10. shows the little difference among ABNC and E-WKCA at the millisecond level which is less than $0.72 \%$.

\section{Conclusion}

This study aims to improve the recent methods in the border cluster problem which may cause the throughput to be suboptimal. Previous methods have approached using the concept of similarity value with the combination of K-Medoids, Genetic Algorithms and Tabu Search. We improve their combination of several algorithms to get a more stable cluster in the border cluster area. Our modifications have never been used before in VANET. We integrate genes without going through the original crossover process. It is a new concept to achieve the best chromosome in a narrow time. The best genes from the parent chromosome are gathered together to build a new cluster. It replaces the traditional crossover schemes. We have evaluated the proposed method and it shows its robustness in the stability of the throughput in varied traffic conditions (speeds, vehicle types and densities). We also compare the results with the recent methods (AKCA and E-WKCA). Although, the recent methods superior to our proposed method, we have better in terms of throughput and cluster size stability. We achieve throughput improvement at 93.97\%. Furthermore, our model can manage the cluster size tend to remain at around 25 vehicles. Our proposed method is competitive when compared to recent methods. In the future, scenarios can be developed further in more complex conditions to achieve a better method.

\section{Acknowledgments}

The work is funded by Research Directorate of Universitas Gadjah Mada through the RTA program 2019 (Contract Number: 3332/UN1/DITLIT/DITLIT/LT/2019).

\section{References}

[1] IEEE, "IEEE Standard for Wireless Access in Vehicular Environments (WAVE) - Networking Services", IEEE Std 16093-2016 (Revision of IEEE Std 16093-2010), pp.1-160, 2016.

[2] R. Adrian, S. Sulistyo, I. W. Mustika, and S. Alam, "A Preliminary Performance Evaluation of Population-Based Algorithms in VANET", In: Proc. of 2019 International Conference of Artificial Intelligence and Information Technology (ICAIIT), pp.220-224, 2019.

[3] X. Duan, Y. Liu, and X. Wang, "SDN Enabled 5GVANET: Adaptive Vehicle Clustering and Beamformed Transmission for Aggregated Traffic", IEEE Communications Magazine, Vol.55, No.7, pp.120-127, 2017.

[4] R. Hajlaoui, E. Alsolami, T. Moulahi, and H. Guyennet, "Construction of a stable vehicular ad hoc network based on hybrid genetic algorithm", Telecommunication Systems, Vol.71, No.3, pp.433445, 2019.

[5] R. S. Bali, N. Kumar, and J. J. P. C. Rodrigues, "Clustering in vehicular ad hoc networks: Taxonomy, challenges and solutions", Vehicular Communications, Vol.1, No. 3, pp.134-152, 2014.

[6] C. Cooper, D. Franklin, M. Ros, F. Safaei, and M. Abolhasan, "A Comparative Survey of VANET Clustering Techniques", IEEE Communications Surveys \& Tutorials, Vol.19, No.1, pp.657-681, 2017. 
[7] R. Hajlaoui, E. Alsolami, T. Moulahi, and H. Guyennet, "An adjusted K-medoids clustering algorithm for effective stability in vehicular ad hoc networks", International Journal of Communication Systems, Vol.32, No.12, pp.1-16, 2019.

[8] R. Adimalla, V. Kumari, and C. Reddy, "Genetic Algorithm based Backup Route Establishment for QoS Routing", International Journal of Intelligent Engineering and Systems, Vol.10, No.3, pp.38-46, Jun. 2017.

[9] G. H. Alsuhli, A. Khattab, and Y. A. Fahmy, "DoubleHead Clustering for Resilient VANETs", Wireless Communications and Mobile Computing, Vol. 2019, pp.1-17, 2019.

[10] R. Adrian, S. Sulistyo, and I. W. Mustika, "A Study on Communication System in VANET", In: Proc of 2018 4th International Conference on Science and Technology (ICST), pp.1-6, 2018.

[11] S. Mirjalili, "Moth-flame optimization algorithm: A novel nature-inspired heuristic paradigm", Knowledge-Based Systems, Vol.89, pp.228-249, 2015.

[12] Y. A. Shah, H. A. Habib, F. Aadil, M. F. Khan, M. Maqsood, and T. Nawaz, "CAMONET: Moth-Flame Optimization (MFO) Based Clustering Algorithm for VANETs", IEEE Access, Vol.6, pp.48611-48624, 2018.

[13] S. Sulistyo, S. Alam, and R. Adrian, "Coalitional Game Theoretical Approach for VANET Clustering to Improve SNR", Journal of Computer Network Communications, Vol.2019, pp.1-13, 2019.

[14] W. Saad, Z. Han, M. Debbah, A. Hjorungnes, and T. Basar, "Coalitional game theory for communication networks", IEEE Signal Processing Magazine, Vol.26, No.5, pp.77-97, 2009.

[15] P. Guo, X. Wang, and Y. Han, "The enhanced genetic algorithms for the optimization design", In: Proc of 2010 3rd International Conference on Biomedical Engineering and Informatics, Vol.7, pp.2990-2994, 2010.

[16] D. de Werra and A. Hertz, "Tabu search techniques", Operation Research Spektrum, Vol.11, No.3, pp.131$141,1989$.

[17] A. M. Chinnaiyan and N. Palanisamy, "Chapter 4 Chromosomal Aberrations in Solid Tumors", In: Progress in Molecular Biology and Translational Science, Vol.95, pp.55-94, 2010,

[18] A. A. Mu'azu, L. T. Jung, I. A. Lawal, and P. A. Shah, "Throughput Measurement for the Guaranteed QoS Real-time Traffic Flows in VANETs", In: Procedia Social and Behavioral Sciences, Vol.129, pp.297-304, 2014.

[19] S. Ayub, S. Kariyawasam, M. Honary, and B. Honary, "A practical approach of VLC architecture for smart city", In: Proc. of 2013 Loughborough Antennas Propagation Conference (LAPC), pp.106-111, 2013. 